BULLETIN OF THE

AMERICAN MATHEMATICAL SOCIETY

Volume 77. Number 6, November 1971

\title{
PRIMITIVE SUBALGEBRAS OF EXCEPTIONAL LIE ALGEBRAS
}

\author{
BY MARTIN GOLUBITSKY AND BRUCE ROTHSCHILD
}

Communicated by S. Sternberg, May 10, 1971

The object of this paper is to classify (up to inner automorphism) the primitive, maximal rank, reductive subalgebras of the (complex) exceptional Lie algebras. By primitive we mean that the subalgebras correspond to (possibly disconnected) maximal Lie subgroups. In [3], the corresponding classification for the (complex) classical Lie algebras was completed, as was the classification for the nonreductive, maximal rank, subalgebras of all the simple Lie algebras.

Using case by case techniques and some more general results given later, we prove the following theorem:

THEOREM 0. The primitive, maximal rank, reductive subalgebras of the exceptional (complex, simple) Lie algebras are listed (up to conjugacy by an inner automorphism) in the table below. Further, all subalgebras isomorphic to one of these are conjugate by an inner automorphism.

Algebra

Primitive subalgebras

$$
\begin{array}{ll}
E_{8} & A_{1} \oplus E_{7}, A_{1}^{8}, A_{2} \oplus E_{6}, A_{2}^{4}, A_{4}^{2}, D_{4}^{2}, D_{8}, A_{8}, T^{8} \\
E_{7} & A_{1} \oplus D_{6}, A_{1}^{3} \oplus D_{4}, A_{1}^{7}, A_{2} \oplus D_{5}, A_{2}^{3} \oplus T^{1}, A_{7} ; E_{6} \oplus T^{1}, T^{7} \\
E_{6} & A_{1} \oplus A_{5}, A_{2}^{3} ; D_{4} \oplus T^{2}, D_{5} \oplus T^{1}, T^{6} \\
F_{4} & A_{1} \oplus C_{3}, A_{2}^{2}, B_{4}, D_{4} \\
G_{2} & A_{1}^{2}, A_{2}
\end{array}
$$

$T^{k}$ denotes the center of the subalgebra, where $k$ is the dimension of that center. The other superscripts refer to the number of summands of the corresponding algebra.

We note that Theorem 5.5 (p. 148) in the reductive case of Dynkin [2] is incorrect. In particular $A_{3} \oplus D_{5}, A_{5} \oplus A_{2} \oplus A_{1}, A_{7} \oplus A_{1}$ in $E_{8}$, $A_{3}^{2} \oplus A_{1}$ in $E_{7}$ and $A_{3} \oplus A_{1}$ in $F_{4}$ are not maximal subalgebras. (See [2, Table 12, p. 155].)

The authors wish to thank Robert Steinberg for several helpful remarks.

We now present some basic notation and a characterization of

AMS 1970 subject classifications. Primary 22E10, 17B20, $17 \mathrm{~B} 25$. 
primitivity from $[3]$.

Let $p$ be a maximal rank subalgebra of a simple Lie algebra $g$. By maximal rank, we mean that there exists a Cartan subalgebra $h$ of $g$ which is contained in $p$. We fix $h$.

Let $W$ be the Weyl group relative to $h . p$ is then decomposed by $h$ into

$$
p=h \oplus \sum_{\phi} e_{\phi}
$$

where the $\phi$ 's are roots in $g$ determined by $h$, and the $e_{\phi}$ 's are the corresponding one-dimensional root spaces. $p$ is then uniquely determined by the roots $\phi$ for which $e_{\phi} \subset p$. Let $K_{p}=\{\phi$ a root of $g$ relative to $\left.h \mid e_{\phi} \subset p\right\}$. Define $W_{p}=\left\{\alpha \in W \mid \alpha\left(K_{p}\right)=K_{p}\right\}$.

Proposition 1.1. Let $p$ be a maximal rank subalgebra of $g$. Then $p$ is primitive iff the following holds: If $l$ is a subalgebra of $g$ such that $p \subset l$, and $W_{p} \subset W_{l}$, then $p=l$ or $l=g$.

Proof. This is Proposition 3.2 of [3].

We will use this as our definition of primitivity.

Now let $p$ be reductive, i.e. $p$ is given uniquely as the direct sum of simple algebras and its center. Thus there exist nonisomorphic simple Lie subalgebras of $g: X_{1}, X_{2}, \cdots, X_{r}$ such that $p=X_{1}^{k_{1}}$ $\oplus \cdots \oplus X_{r}^{k_{r}} \oplus T$ where $T$ is the center of $p$ and where $X_{i}^{k_{i}}$ denotes the direct sum of all ideals of $p$ isomorphic to $X_{i}$, and $k_{i}$ is the number of such ideals. Note that since $X_{i}=\left(h \cap X_{i}\right) \oplus \sum_{\phi} e_{\phi}$, where the $\phi$ are unique, $K_{X_{i}}$ and $W_{X_{i}}$ make sense.

Lemma 1.2. Let $q_{i}=X_{i}^{k_{i}}$. Then $W_{p} \subset W_{q_{i}}$.

Let $z$ be a subalgebra of $g$ with Cartan subalgebra $h_{z} \subset h$. Assume that $z$ is regular (in the sense of Dynkin [2]), i.e. let $K_{z}=\left\{\phi \in K_{\theta} \mid e_{\phi} \subset z\right\}$, then $z=h_{z} \oplus \sum_{\phi \in K_{z}} e_{\phi}$. Denote by $K_{z}^{\perp}$ all of the roots in $K_{g}$ orthogonal to the set $K_{z}$. Let $h_{z}^{\perp}$ be the subspace of $h$ orthogonal to $h_{z}$.

Lemma 1.3. Let ${ }_{z}^{\perp}=h_{z}^{\perp} \oplus \sum_{\phi \in K_{z}^{\perp}} e_{\phi}$. Then $z^{\perp}$ is a subalgebra of $g$, and $K_{z}^{\perp}=K_{z}^{\perp}$.

TheOREM 1.4. Let $p$ be a maximal rank, reductive subalgebra. Let $p=X_{1}^{k_{1}} \oplus \cdots \oplus X_{r}^{k_{r}} \oplus T$ (as described above). Let $q_{i}=X_{i}^{k_{i}}$. If $p$ is primitive, then either $q_{1}^{\perp}=q_{2} \oplus \cdots \oplus q_{r}$ or the subalgebra, generated by the vector subspace $q_{1}+q_{1}^{\perp}+h$ is $g$.

CoROLLARY 1.5. Let $g$ be a simple Lie algebra all of whose roots have the same length (in particular $E_{6}, E_{7}$, and $E_{8}$ ). Then if $p$ is a primitive subalgebra, $Y=X_{2}^{k_{2}} \oplus \cdots \oplus X_{r}^{k_{r}}$. 
To compute the primitive subalgebras we use a case by case technique. For each exceptional algebra we determine which simple algebras could be ideals in a maximal rank, reductive subalgebra. The possibilities are restricted by rank and the length of the roots. For instance, the only possibilities for $X_{1}$ in $E_{8}$ are $A_{l}(1 \leqq l \leqq 8)$, $D_{l}(4 \leqq l \leqq 8), E_{6}$ and $E_{7}$. Then using 1.4 and 1.5 , we know what the possibilities are for the subalgebra. For example, in $E_{8}$, given $X_{1}^{k_{1}}$, the only possibility for a primitive subalgebra is $X_{1}^{k_{1}} \oplus\left(X_{1}^{k_{1}}\right)^{\perp}$ by 1.5 .

The remaining task is to determine whether or not the given subalgebra is primitive. For this we use Proposition 1.1. Unfortunately (particularly for $\left.E_{s}, s=6,7,8\right)$ this is a nontrivial task. We need to know more about the Weyl group of $E_{s}$. We present here a theorem which we found extremely useful in computing with these Weyl groups.

We first describe the roots of the algebras $\mathrm{E}_{6}, E_{7}$, and $E_{8}$ (see [1]). Let $z_{1}, z_{2}, \cdots, z_{8}$ be the standard orthonormal basis for the dual space to a fixed Cartan subalgebra of $E_{8}$. With respect to this basis, the roots of $E_{8}$ are given by

$$
(\mathrm{I})_{8}=\left\{ \pm z_{i} \pm z_{j} \mid 1 \leqq i<j \leqq 8\right\}
$$

and

$(\mathrm{II})_{8}=\left\{\frac{1}{2}\left( \pm z_{1} \pm z_{2} \pm \cdots \pm z_{8}\right) \mid\right.$ the number of minus signs is even. $\}$.

We shall refer to these as type I roots and type II roots respectively.

We take $E_{6}$ and $E_{7}$ to be regular subalgebras of $E_{8}$. The roots of $E_{7}$ are all of those roots of $E_{8}$ orthogonal to $z_{7}+z_{8}$. Thus

$$
\begin{aligned}
(\mathrm{I})_{7} & =(\mathrm{I}) \cap K_{E_{7}}=\left\{ \pm z_{i} \pm z_{j} \mid 1 \leqq i<j \leqq 6\right\} \cup\left\{z_{7}-z_{8}\right\}, \\
(\mathrm{II})_{7} & =(\mathrm{II}) \cap K_{E_{7}}=\left\{ \pm \frac{1}{2}\left(z_{1} \pm z_{2} \pm \cdots \pm z_{6}+z_{7}-z_{8}\right) \in(\mathrm{II})_{8}\right\} .
\end{aligned}
$$

The roots of $E_{6} \subseteq E_{7}$ are as follows:

$$
\begin{aligned}
(\mathrm{I})_{6} & =(\mathrm{I}) \cap K_{E_{6}}=\left\{ \pm z_{i} \pm z_{j} \mid 1 \leqq j<j \leqq 5\right\}, \\
(\mathrm{III})_{6} & =(\mathrm{II}) \cap K_{E_{6}} \\
& =\left\{ \pm \frac{1}{2}\left(z_{1} \pm z_{2} \pm \cdots \pm z_{5}+z_{6}+z_{7}-z_{8}\right) \in(\mathrm{II})_{7}\right\} .
\end{aligned}
$$

We note first that the reflections about roots of type $I$ are just determined by signed permutations of $z_{1}, \cdots, z_{8}$. For if $\alpha$ and $\beta$ are roots, then

$$
S_{\alpha}(\beta)=\beta-\frac{2(\alpha, \beta)}{(\alpha, \alpha)} \alpha=\beta-(\alpha, \beta) \alpha, \quad \text { as }(\alpha, \alpha)=2 .
$$


If $\alpha=z_{i}-z_{j}$, then

$$
\begin{aligned}
S_{\alpha}\left(z_{k}\right) & =z_{i} & & \text { if } k=j, \\
& =z_{j} & & \text { if } k=i, \\
& =z_{k} & & \text { otherwise. }
\end{aligned}
$$

If $\alpha=z_{i}+z_{j}$, then

$$
\begin{aligned}
S_{\alpha}\left(z_{k}\right) & =-z_{i} & & \text { if } k=j \\
& =-z_{j} & & \text { if } k=i, \\
& =z_{k} & & \text { otherwise. }
\end{aligned}
$$

We can thus, for example, identify $S_{\alpha}$ with the transposition

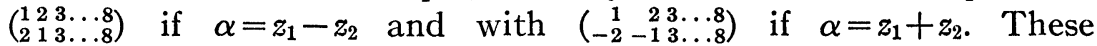
reflections, then, generate signed permutations with an even number of sign changes.

Let $N_{s}$ be the subgroup of the Weyl group of $E_{s}$ consisting of the signed permutations. We have the following normal forms for elements of the Weyl group:

TheOREM 1.6. Let $g$ be a Weyl group element. Then one of the following three cases holds:

(a) $g \in N_{s}$.

(b) $\exists f \in N_{s}, \alpha \in(\mathrm{II})_{s} \ni g=f S_{\alpha}$.

(c) $\exists f \in N_{s}, \alpha, \beta \in(\mathrm{II})_{s} \ni(\alpha, \beta)=0$ and $g=f S_{\beta} S_{\alpha}$.

\section{BIBLIOGRAPHY}

1. N. Bourbaki, Groups et algèbres de Lie. Chaps. 4, 5, 6, Actualités Sci. Indust., no. 1337, Hermann, Paris, 1968. MR 39 \#1590.

2. E. B. Dynkin, Semisimple subalgebras of semisimple Lie algebras, Mat. Sb. 30 (72) (1952), 349-462; English transl., Amer. Math. Soc. Transl. (2) 6 (1957), 111-244. MR 13, 904.

3. M. Golubitsky, Primitive actions and maximal subgroups of Lie groups, J. Differential Geometry (to appear).

University of California, Los Angeles, California 90024

Current address: (Martin Golubitsky) Massachusetts Institute of Technology, Cambridge, Massachusetts 02139 